Brit. J. Ophthal. (1960) 44, 606.

\title{
CHANGES IN THE ELECTRO-OCULOGRAM POTENTIAL LEVEL*
}

\author{
BY \\ JEAN R. DAVIS AND B. SHACKEL \\ Psychological Research Laboratory, EMI Electronics Ltd., Hayes, Middlesex
}

THE eyeball may be regarded as a small battery, with a positive pole in the cornea and a negative pole in the retina. The work of Fenn and Hursh (1937), François, Verriest, and de Rouck (1957), and others, suggests that the corneo-retinal potential is associated primarily with the retinal receptors; Brindley (1956) has suggested that the resting potential of the lens may contribute to it, and it may be that the cornea also contributes. Changes in the position of the eyeball cause changes in potential at the skin surface round the eye socket; these changes can be detected, amplified, and measured by the technique known as electro-oculography (EOG). The changes of potential are, for practical purposes, linearly related to the angle of the eyeball rotation and have been used as measures of eye movement and eye position. Conversely, by having the subject make known angular rotations of the eyeball, the potential changes can be recorded under standard conditions to give the "EOG potential level".

Provided that the measurement conditions are standardized and that the body structure surrounding the eyeball is a relatively homogeneous conducting medium, at least in the plane of movement, the "EOG potential level" may be considered as a direct function of, and therefore as a measure of, the potential difference between cornea and retina. The following definitions have therefore been suggested (Shackel, 1960):

The corneo-retinal standing potential is the actual potential difference between cornea and foveal sclera as measured by electrodes placed in contact with those structures, and thus is normally measurable exactly only on freshly excised eyes.

The EOG potential is the corneo-retinal standing potential as measured in an EOG situation by means of peri-orbital electrodes suitably placed to record a potential difference when the eyeball position in the plane of the electrodes departs from the mean zero point (at which cornea and central retina are approximately equidistant from the electrodes).

* Received for publication October 20, 1959. 
The EOG potential level of a subject is a measure of the corneo-retinal standing potential level for that subject, because it is the EOG potential recorded under standard conditions.

The EOG potential level at any time is the mean potential recorded for a $30^{\circ}$ excursion; it is the mean of at least one excursion each side of centre to $30^{\circ}$ left and $30^{\circ}$ right; it may be expressed simply in millivolts or microvolts, the standard conditions being assumed, or may be divided by 30 and expressed in microvolts per degree. Unless stated to be uniocular, it is assumed that any EOG potential level is a binocular level (because Lead 3, as defined by Miles, horizontally across both eyes gives more reliable results; Miles, 1939a).

Large differences in EOG potential level between subjects have been found by all workers (Fenn and Hursh, 1937; Miles, 1939a, b; Leksell, 1939; Mackensen and Harder, 1954; François and others, 1957; Shackel, 1960). Fenn and Hursh (1937) reported constancy of potential level for individuals over time, but subsequent workers (Miles, 1939b; Mackensen and Harder, 1954; Kris, 1959) have reported significant differences in the same individuals on different occasions. In two surveys on approximately 100 subjects, separated by an interval of 10 months, test-retest correlations of $r=+0 \cdot 66$, uncorrected for differences in time of testing during the day, and of $r=+0.83$, for subjects tested at the same time, within the hour, on the two occasions, were found; these suggest considerable constancy of potential level with time (Shackel, 1960; Shackel and Davis, 1960).

Miles (1939c), in studying the effects of experimental modification on the potential, found marked changes under certain conditions. He suggested that potential changes conformed to a pattern similar to that of blood pressure, metabolism, and other factors which decrease with relaxation and inactivity and increase with tension and apprehension.

However, no reports have been found of prolonged studies of the EOG potential level in the same group of subjects under controlled conditions of measurement.

\section{Purpose}

The experiment was planned with the following aims:

(1) To study the variations in EOG potential levels recorded at the same time of day, from Monday to Friday, for 8 weeks, under controlled conditions;

(2) To study the changes from hour to hour in subjects tested throughout a working day;

(3) At the same time to gather further information about the subjects, both objectively and subjectively, with which to correlate any variations in EOG levels. 


\section{Apparatus}

A portable version of the electronic apparatus developed for measuring EOG (Shackel, Sloan, and Warr, 1958) was used. The positions of the rubber suction cup electrodes (Shackel, 1958) were determined by a simple perspex jig (Fig. 1), which was placed against the outer canthus of the eye with the arrow in line with the line through the centre of the two pupils. The jig was then rotated sideways to press flat against the facial skin, the arrow still being in line with the pupils. Marks were made with a skin pencil through the holes in the jig to give the exact position for the electrodes.

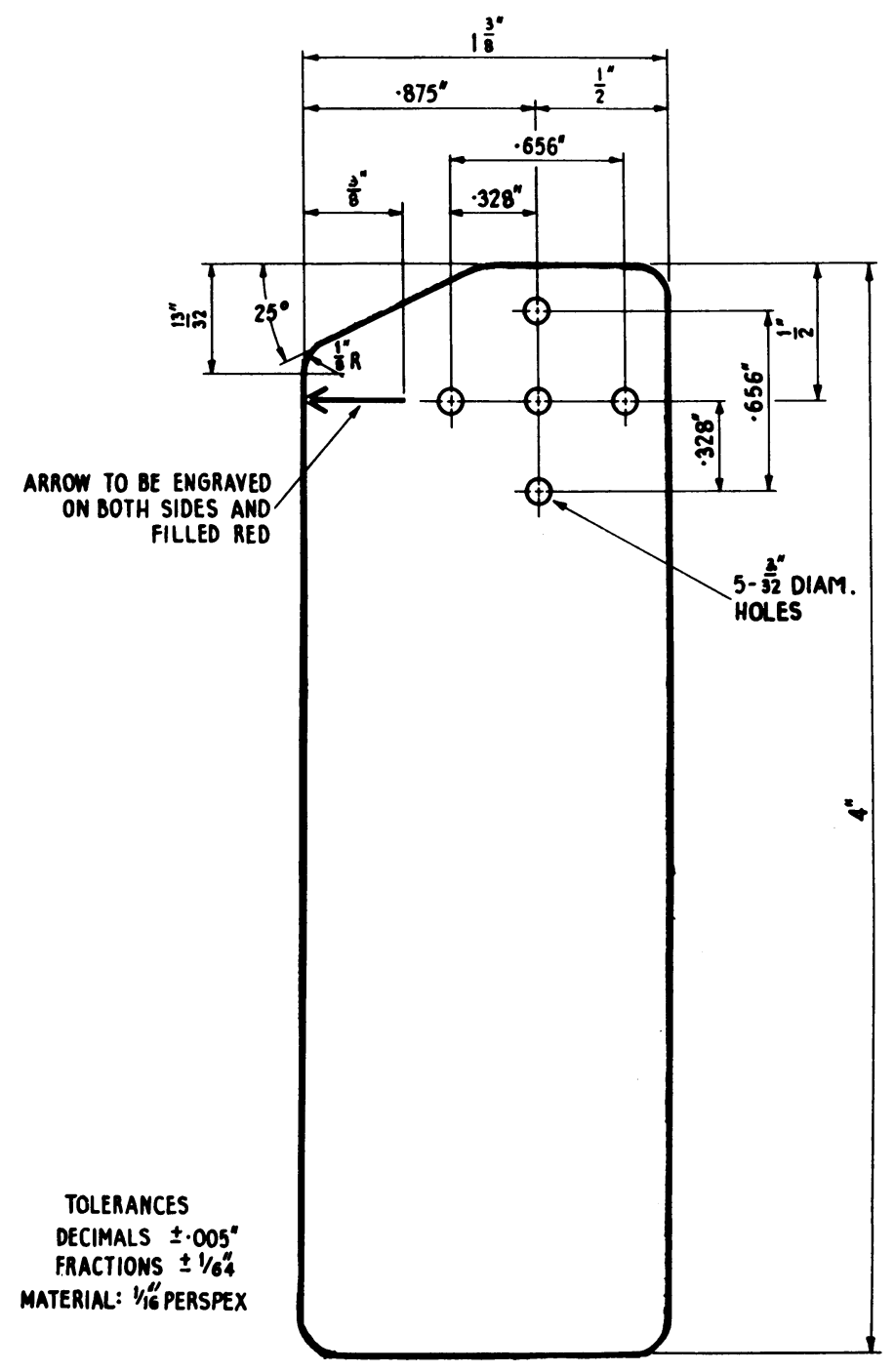

FIG. 1.-Electrode siting jig. 
The target apparatus consisted of a T-shaped piece of white board mounted at a distance of 16 in. from a clamp, into which a wooden spatula was inserted to serve as a biting bar. The distance of each subject's eyes from the target when biting on to the spatula was checked by means of a measuring stick to be exactly 15 in. The target covered the central area of the visual field, extending $60^{\circ}$ horizontally and $35^{\circ}$ vertically; it was illuminated by two 100 -watt bulbs and had a mean brightness of 700 foot-lamberts. On the target board were four light grey fixation points: a central zero, fixation points to the left and right of this which gave exactly $30^{\circ}$ of eye movement, and a fourth point which was used as a reference centre during adaptation. The target apparatus had two handles and a foot so that each subject could lift or rest it in a comfortable position. The subject was seated at a table on which the target was placed. The experimenter sat at the same table with the electronic apparatus, on which the potential levels were recorded by a meter (Fig. 2).

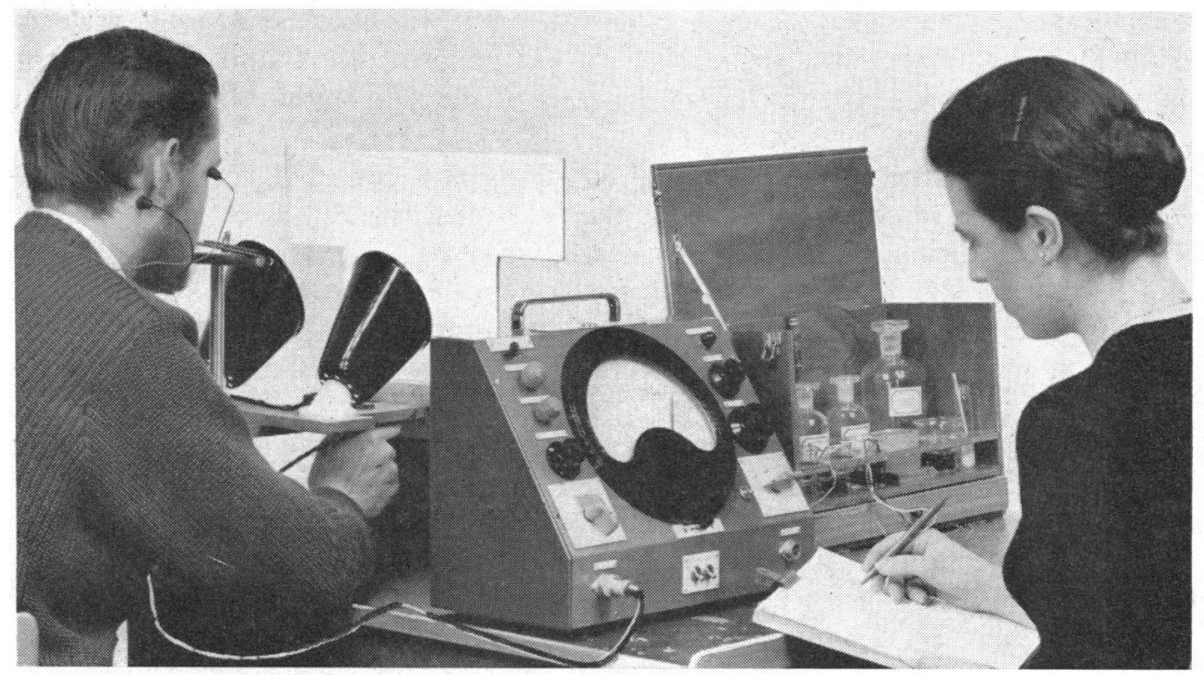

FIG. 2.-Subject, target, electronic apparatus, electrode box, experimenter.

The record sheets were in the form of a questionnaire which the subjects completed each day, stating how many hours they had slept, whether they felt tired or unwell, if they had had any alcohol the night before, their time of rising that morning, and similar questions. Below the questions were spaces for the experimenter to fill in the subject's temperature, pulse, and potential readings.

\section{Subjects}

There were twelve subjects, six male and six female. The subjects were of two age groups $20-25$ years and $40-45$ years, three male and three female in each group, and within these groups were approximately equated for intelligence and occupational status. The two experimenters also acted as subjects, giving a total of fourteen. 


\section{Method}

The subject sat down, and the skin at the sides of the eyes and behind the right ear, where the electrodes were to be placed, was cleaned with acetone. The positions of the electrodes were marked with the jig; the electrodes were filled with 1 per cent. saline jelly and applied. Because each reading of potential would take a very short time, and zero drifts were therefore not expected to be obtrusive, the skin drill (Shackel, 1959) was not used; it was considered that no significant errors would arise, in reading the meter, from estimating corrections for any small drifts that might occur.

While the subject was being prepared for testing he completed the questionnaire. The subject then sat directly in front of the target, but not biting the spatula, and adapted to the illumination level for $4 \mathrm{~min}$. (a time found to be the optimum by previous testing). During this time his temperature was recorded and his pulse taken. During the fourth minute the subject was asked to bite on to the spatula, and the exact distance of the eyeball from the target was checked with the measuring stick. The subject was then asked to fix the zero point, and the recording apparatus was set to zero, so that at the start of the fifth minute the first reading could be made.

The testing routine consisted of $0-\mathrm{L} 30^{\circ}-0-\mathrm{R} 30^{\circ}-0$ repeated four times at as constant intervals as possible. After the last measure had been recorded the subject ceased biting, the electrodes were removed, and the skin was wiped clean of saline jelly. The spatula was replaced in readiness for the next subject. The whole testing procedure took $8 \mathrm{~min}$. and the subjects reported at 10-min. intervals at the same time every working day for 8 weeks. All tests were done between 8.30 and $10.50 \mathrm{a} . \mathrm{m}$.

The time of $4 \mathrm{~min}$. for adaptation to the target brightness, the level of which was fairly similar to the surrounding room, was arrived at as a result of a pilot study on four subjects. Readings were taken every minute for up to $10 \mathrm{~min}$. after switching on the lights. It was found that by the end of $4 \mathrm{~min}$. an approximate plateau of potential level had been reached.

Although Mackensen and Harder (1954) reported that at least five separate excursions to left and right are necessary to give a reliable mean EOG level, only four readings were used here on the basis of an analysis of data available in the laboratory. These data consisted of a sample of fifty sets of groups of five readings to left and to right. The means of the groups of five were computed and compared with the means of the first four readings of the groups of five; no difference between these means was greater than $2 \mu \mathrm{V}$. It was felt therefore that four readings to left and right would give a reliable estimate of the true potential level.

In order to check that slight differences in head position, or in amounts of eye closure, would not significantly affect the results, a study was done in which the subjects were tested under experimental conditions with considerable degrees of head tilt and eye closure. None of these positions yielded a significant difference from the normal potential level of the subjects.

It is considered that experimental error resulting from apparatus, estimation of drift, or minor changes in electrode or head position cannot have exceeded $\pm 25 \mu \mathrm{V}$; taking a very conservative view the possible experimental error may be safely regarded as less than $\pm 50 \mu \mathrm{V}$. 


\section{Results}

\section{Stability and Variability of Daily and Weekly Levels}

The results support the work of Fenn and Hursh (1937), Miles (1939a, b), Leksell (1939), Mackensen and Harder (1954), and François and others (1957), in showing very large differences in potential levels between subjects. The individual daily scores ranged from 255 to $1,110 \mu \mathrm{V}$, and Fig. 3, showing the mean weekly potential level for each subject, illustrates clearly the wide range of potential levels found in this group of subjects. The grand mean for the group was $713 \mu \mathrm{V}$.

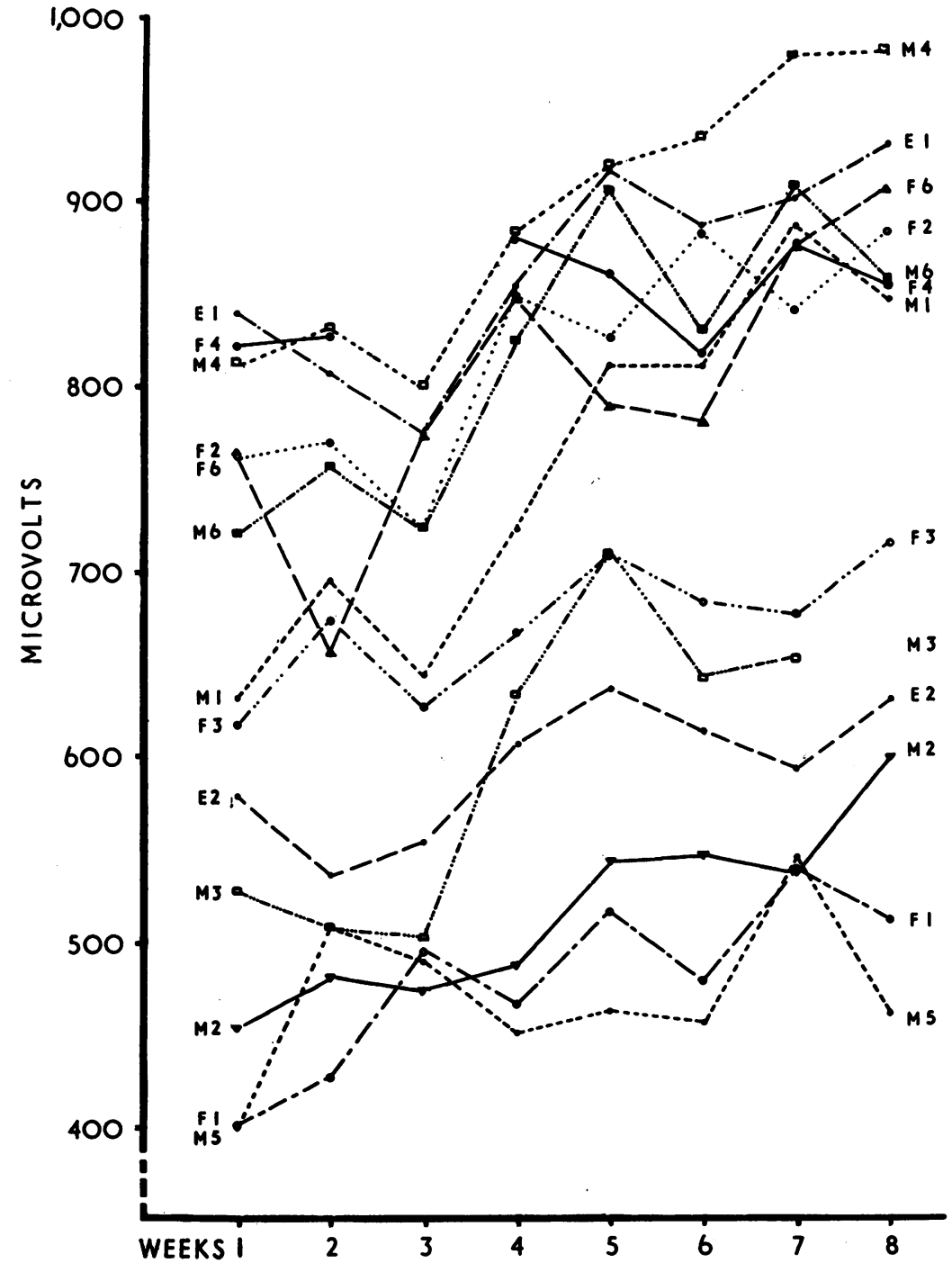

FIG. 3.-Mean weekly EOG level (results of F5 not shown because of absence for Weeks 4, 5, 6). 
This mean level is higher than those of 580 and $562 \mu \mathrm{V}$ found in two surveys of 100 young male subjects (Shackel, 1960; Shackel and Davis, 1960). Other experimenters have reported different mean levels for groups of subjects, for instance Miles (1939a) reported a mean level of more than $1 \mathrm{mV}$ from 56 female subjects. Further experimentation is needed to find out if these differences are artefacts of sampling, or are caused by climatic or racial differences, or, as is most likely, by different positioning of electrodes.

(a) Stability.-Although the distribution of potential levels for different individuals is very large, it was found that for each individual the changes in potential level from week to week were quite small. The average change in level was only $46 \mu \mathrm{V}$ (S.D. $=31 \mu \mathrm{V})$, and 95 per cent. of the changes were less than $105 \mu \mathrm{V}$ (Fig. 4).

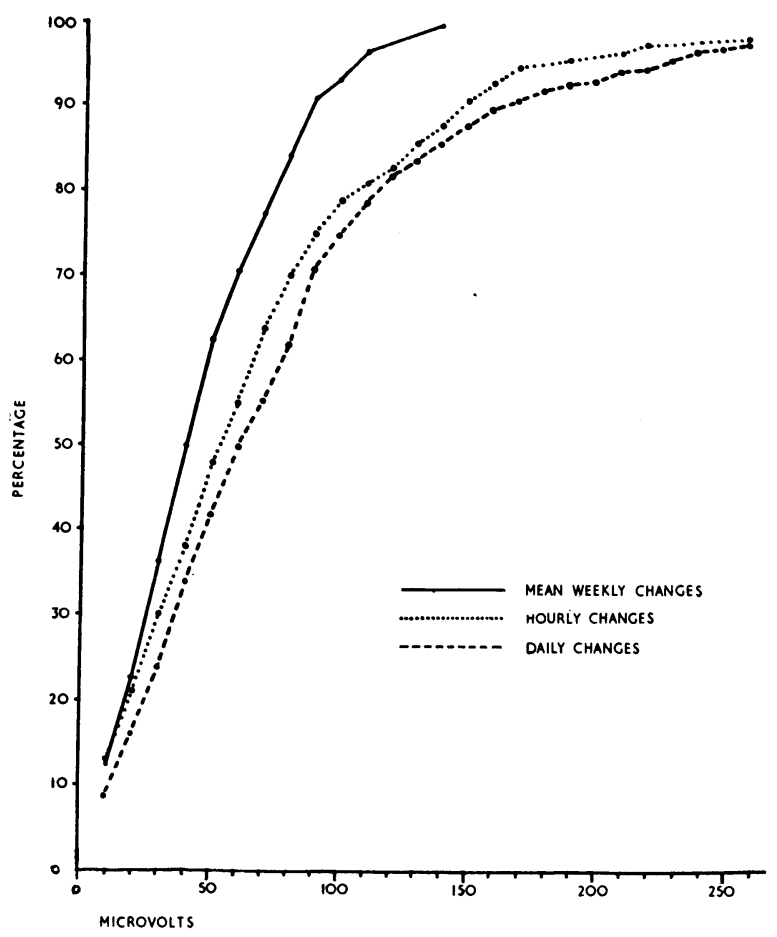

FIG. 4.-Percentage of hourly, daily, and mean weekly changes in EOG potential level of less than given abscissa value.

The mean EOG level for each week was calculated for each subject, and a rank-order correlation coefficient $(\rho)$ was determined for each week against the first week as follows:

$\begin{array}{llll}\text { Weeks } 1 \text { and } 2 & \rho=+0.92 & \text { Weeks } 1 \text { and } 5 & \rho=+0.91 \\ \text { Weeks } 1 \text { and } 3 & \rho=+0.97 & \text { Weeks } 1 \text { and } 6 & \rho=+0.91 \\ \text { Weeks } 1 \text { and } 4 & \rho=+0.97 & \text { Weeks } 1 \text { and } 7 & \rho=+0.84 \\ & \text { Weeks } 1 \text { and } 8 & \rho=+0.91\end{array}$


As the lowest $\rho$ of +0.84 (for Weeks 1 and 7 ) is still very significant, it is obvious that the mean potential levels are relatively stable. As a further check the $\rho$ values were calculated between the grand mean and the means of the first and last weeks and yielded values of $\rho=+0.96$ and $\rho=+0.95$ respectively. It seems then that over the 8 weeks of the experiment the mean potential level for each subject, with relation to that of the other subjects, remained very constant and could almost be regarded as characteristic of that person.

(b) Variability.-This consistency is only apparent in the mean weekly levels; the day by day scores do not, for the majority of subjects, show the same constancy. Some subjects gave large changes from one day to the next on occasions, the largest being $400 \mu \mathrm{V}$. The average daily change in level was $76 \mu \mathrm{V}($ S.D. $=65 \mu \mathrm{V}), 6$ per cent. of the changes being greater than $200 \mu \mathrm{V}$ and 25 per cent. greater than $100 \mu \mathrm{V}$ (Fig. 4).

As experimental error was certainly less than $\pm 50 \mu \mathrm{V}$, another explanation for these changes must be sought. It is interesting, in view of these large fluctuations from day to day, that the mean level should remain so constant. However this paradox would explain the differences in the literature, some workers regarding EOG as a stable measure and others as unstable.

\section{Diurnal and Nocturnal Levels}

(a) Diurnal Changes.-Kris (1959) reported a diurnal pattern in changes of EOG potential, with a day-time peak at about 12.0 noon to $2.0 \mathrm{p} . \mathrm{m}$. and a trough in the night or very early morning, broadly corresponding to the diurnal pattern of metabolic activity. In the present experiment, subjects were tested at hourly intervals through a working day, giving eight recordings for each subject. The range of variation over a day for the subjects was very wide; some few showed variations up to $300 \mu \mathrm{V}$, whereas others were relatively stable for the whole day. The average hourly change in level was $67 \mu \mathrm{V}$ (S.D. $=58 \mu \mathrm{V}$ ), and the distribution was similar to the distribution of daily changes, with 3 per cent. greater than $200 \mu \mathrm{V}$ and 21 per cent. greater than $100 \mu \mathrm{V}$ (Fig. 4).

Concerning the pattern of changes, five of the fourteen subjects tested were so stable that no conclusions could be drawn about diurnal pattern; none of their hourly changes exceeded $50 \mu \mathrm{V}$ (i.e. $\pm 25 \mu \mathrm{V}$, the estimate of probable experimental error). The curves given by the other nine subjects could be broadly grouped into three sets of patterns. The largest group (five subjects) showed a peak at 11.0 a.m., a trough at 2.0 p.m., and a rise at 5.0 p.m. The second pattern (two subjects) showed a rise at 12.0 noon, followed by a steep drop to 1.0 p.m., with an equally sharp rise at 2.0 p.m. The third pattern (two subjects) showed a relatively stable level until a sharp drop at 3.0 p.m. None of these patterns corresponds to that found by Kris. It would seem therefore that there is no definite pattern of diurnal change in potential level common to all persons. 
(b) Nocturnal Changes.-Two additional subjects were tested at hourly intervals during the night from $7.0 \mathrm{p} . \mathrm{m}$. to $7.0 \mathrm{a} . \mathrm{m}$. Both subjects showed a wide range of potential level, with marked fluctuations from hour to hour. The patterns of changes for these two subjects were markedly similar, in spite of differences in times and amounts of food intake, exercise, etc. (Fig. 5).

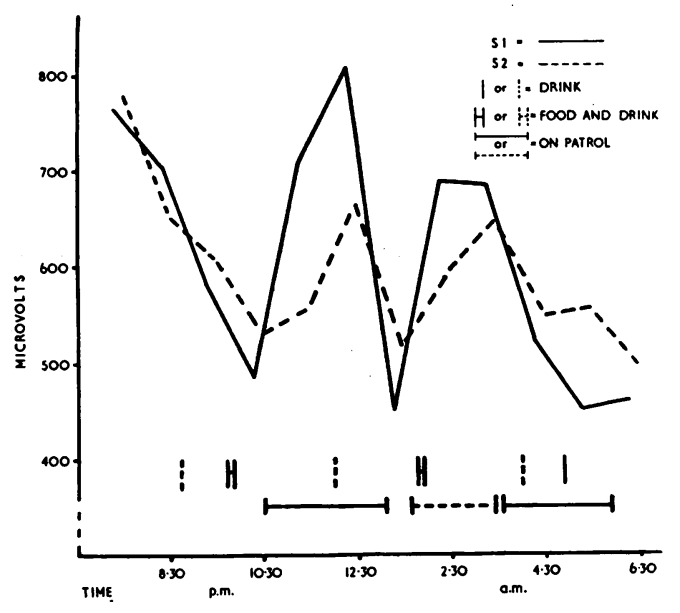

Fig. 5.-All-night test, two subjects.

Moreover, for one subject, this was his first night duty after four nights' rest, while for the other this was his third night on duty. The experimenters who were tested at the same time did not exhibit these changes (Fig. 6).

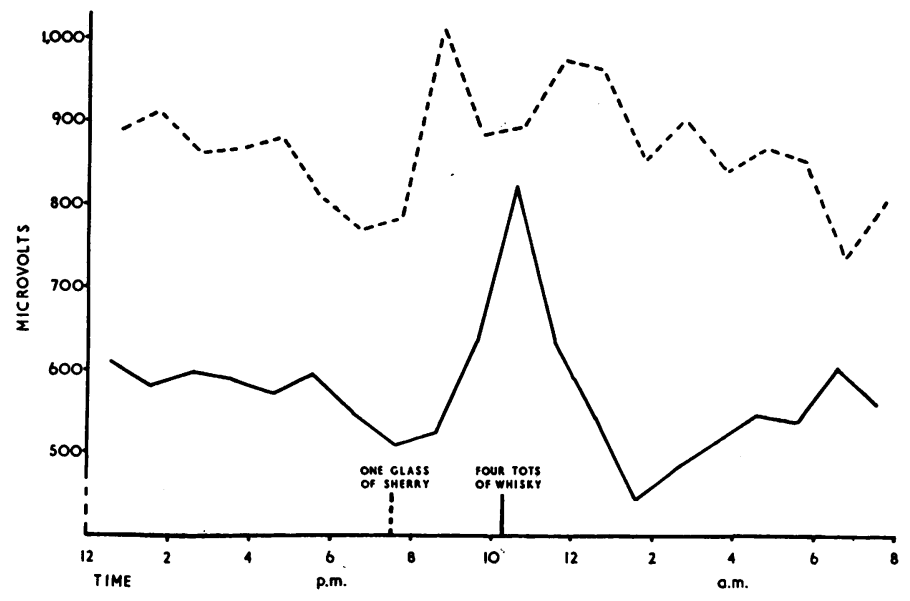

FIG. 6.-20-hour test, two subjects.

Although the subjects, unlike the experimenters, were used to working at nights and had rested during the previous day, these factors do not seem to account for the differences in pattern. The experimenters' peak potential levels seem to be as inexplicable as the subjects', except that some alcohol 
was consumed shortly beforehand by the experimenters, which may or may not be relevant.

\section{EOG and other Factors}

(a) Age and Sex.-Previous studies (Miles, 1939a; Shackel and Davis, 1960) have shown that the EOG potential level tends to decrease with increase in age. In this experiment the mean potential level of the older group (4045 yrs) was $781 \mu \mathrm{V}(\sigma=141 \mu \mathrm{V} ; N=6)$, and that of the younger group (20-25 yrs) was $639 \mu \mathrm{V}(\sigma=123 \mu \mathrm{V} ; N=6)$. This difference, although large, is not significant.

The means and standard deviations of the changes in potential level between consecutive days were computed for each subject. Comparisons were then made between the age and sex of the subjects and their mean change in potential level; there was no significant difference between the male and female groups, but there was a significant rank-order correlation between increasing age and larger mean change in level $(\rho=+0 \cdot 70 ; t=3 \cdot 38 ; N=12 ; P=<0.01)$.

In order to obtain a measure, independent of the absolute size of the mean and standard deviation, for comparing the variability of their changes in level with the age and sex of the subjects, the coefficient of variation $V$, i.e. $\left(\frac{100 \sigma}{\bar{X}}\right)$, was calculated for each subject. There was then found to be a significant rank-order correlation between increasing age and increasing $V(\rho=+0 \cdot 78$; $t=4 \cdot 26 ; N=12 ; P=0.002)$. By using the method of Whitfield (1947), a significant tendency was also found for the male group of subjects to have a higher $V$ than the female group $(\tau=+0.40 ; P=0.05)$.

Thus it is clear that, regardless of the amplitude of their EOG potential level, which was not found to be significantly correlated with age, the older subjects show both larger changes in their EOG level from day to day and greater variability in amplitude of changes from day to day than do the younger subjects. To a less extent the male subjects show greater variability in amplitude of changes than do the females.

(b) Atmospheric Conditions.-An unexpected result was that, for all fourteen subjects, there was a marked tendency for the mean weekly EOG level to rise through the 8 weeks (see Fig. 3). This rise occurred in varying degree with every subject and was quite independent of individual voltage levels.

Miles (1939a) has shown that the potential tends to diminish with relaxation and habituation, so that this increase cannot be explained as adaptation to the experimental situation. Barnett (1940) showed that the resistance of the skin fluctuates seasonally and is diminished during October to December (the time of our experiment). However, the decrease which he reports seems too small to have affected the EOG potential levels, because the recording apparatus had an input impedance of more than two megohms. 
The graph given by the weekly mean EOG levels for the whole group was compared with that for the weekly mean atmospheric temperatures recorded at Kew, 6 miles from the laboratory (Fig. 7), and it was noted that both graphs had two markedly different levels. In the first 3 weeks the temperature was fairly high and the potential level fairly low. In the last 3 weeks the temperature was lower and the voltage correspondingly higher. This apparent correlation may of course only be incidental and not causal.

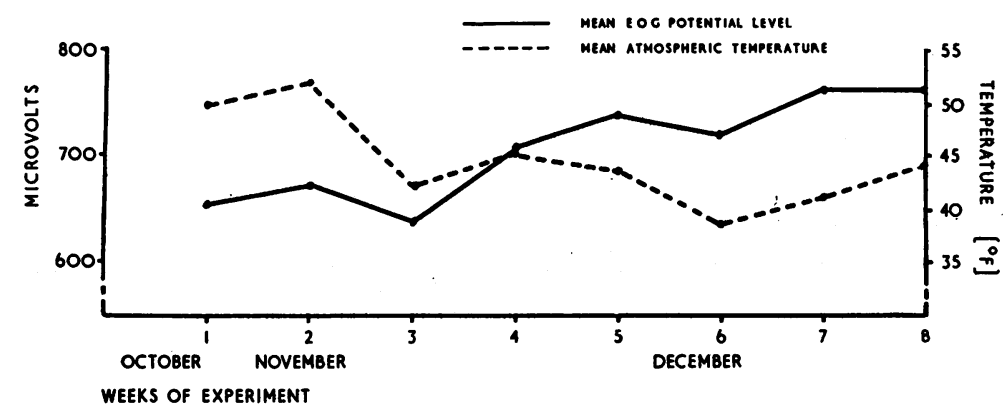

Fig. 7.-Total group mean weekly EOG level compared with mean weekly atmospheric temperature.

Sweeney, Kinney, and Ryan (1960) have recently reported a study in which the scotopic sensitivity of three subjects was found to be poorest in the summer months and to increase gradually in the autumn and winter; the course of the changes in sensitivity agreed well with the two measures taken of previous exposure to sunlight. Large changes in the present and immediately preceding level of ambient illumination are known to influence the EOG potential level (Miles, 1940; ten Doesschate and ten Doesschate, 1956; François and others, 1957; Kris, 1958). One might perhaps speculate upon the possibility of a partial dependence of the EOG potential level, not so much upon ambient temperature, but rather upon the amount of previous exposure of the subject to sunlight.

(c) Metabolic Factors.-A relationship has been suggested between EOG and metabolic factors (Miles, 1939c), because the potential level tends to be higher when the subjects are apprehensive and to diminish with relaxation and inactivity. The results of the present experiment are conflicting. Du Bois (1936) quotes evidence for a correlation of pulse rate and temperature with BMR. No correlation was found in this experiment between the relatively narrow range of temperatures, and pulse rates, and the EOG potential level; however, on two occasions, a drop of over $200 \mu \mathrm{V}$ was accompanied by a drop in temperature and subsequent illness and absence from work. Another factor which has been shown to affect basal metabolism, lack of sleep (Kleitman, 1939; Du Bois, 1936), was compared with potential level, but yielded no significant correlation. 
Osiba (1957) has shown that BMR increases in step with decreases of atmospheric temperature in the winter months, provided the subjects do not live continually in a centrally-heated environment (a proviso satisfied in the case of our subjects). If, therefore, the apparent seasonal variation in EOG potential level is mediated by atmospheric temperature, it may further be possible that the EOG potential level depends in some way upon the BMR and is related to metabolic factors.

It would seem that, if there is a relationship between EOG potential level and metabolic factors, the relationship may not be clearcut and may be influenced by other factors. On the other hand it may be that the changes in potential level and in metabolic correlates recorded in this experiment were small and relatively random because the subjects were "normal". It is possible that in clinical states much larger, correlated, differences may be found. However, a brief test on three thyrotoxic patients has also not yielded any very positive evidence.

\section{Conclusions}

This experiment has satisfactorily fulfilled its primary purpose of providing adequately controlled statistical evidence upon the variability of the EOG potential level. It has shown that moderate constancy, found previously between two occasions 10 months apart, is maintained also between mean weekly levels, but that considerable changes occur in hourly and daily levels. Caution, in the form of moderately frequent calibration, is therefore indicated when EOG is used as a routine technique for recording eye movements. The secondary purpose, to search for relevant correlations of EOG with other variables, has been fulfilled negatively for the most part. The explanation of the correlations which have been found, and also of the paradoxical stability and variability, remains, like many other factors about the EOG potential, to be elucidated.

\section{Summary}

(1) The EOG potential levels, pulse rate, oral temperature, and subjective estimates of tiredness, amount of sleep, etc. of fourteen subjects were recorded on each working day for 8 weeks. Each subject was also tested at hourly intervals throughout one day, two subjects were tested throughout one night, and two subjects throughout 20 hours.

(2) The mean potential levels for all subjects were found to be fairly constant $(\rho=+0.91$ between the means of Week 1 and Week 8$)$ and seem in some way to be characteristic for each individual. Despite this consistency over a long time, there is considerable fluctuation about the mean level from day to day, changes as large as 30 to 50 per cent of the mean level being recorded for some subjects. 
(3) Statistics of the hourly, daily, and mean weekly changes in level are presented. Several patterns for diurnal hourly changes of EOG were found and also an apparent nocturnal pattern for two subjects.

(4) Regardless of the amplitude of their EOG potential level, which was not found to be significantly correlated with age, the older subjects showed both larger changes in their EOG level from day to day and greater variability in amplitude of changes from day to day than the younger subjects. To a less extent the male subjects showed greater variability in amplitude of changes than the female.

(5) No significant correlations were found with metabolic variables, but a definite rise in EOG potential level between the beginning and the end of the experiment may be related in some way to a change in the seasonal atmospheric temperature.

(6) The causes of the stability and of the changes in the EOG potential level are still uncertain.

We would thank the subjects for their conscientious co-operation throughout the prolonged testing schedule, Dr. E. E. Pochin for assistance and permission to make brief tests at University College Hospital, London, the Air Ministry for data on outdoor temperatures at Kew, and the Directors of E.M.I. Electronics Ltd. for encouragement in this work and permission to publish the results.

\section{REFERENCES}

Barnett, A. (1940). Amer. J. Physiol., 129, 306.

BRINDley, G. S. (1956). Brit. J. Ophthal., 40, 385.

Du BoIs, E. F. (1936). "Basal Metabolism in Health and Disease", 3rd ed. Baillière, Tindall \& Cox, London.

Fenn, W. O., and Hursh, J. B. (1937). Amer. J. Physiol., 118, 8.

FrançoIs, J., Verriest, G., and DÉ Rouck, A. (1957). Fortschr. Augenheilk., 7, 1.

Kleitman, N. (1939). "Sleep and Wakefulness". University of Chicago Press, Chicago.

KRIS, C. (1958). Nature (Lond.), 182, 1027.

(1959). "Proc. XV Int. Congr. Psychol. Brussels, 1957”, Acta psychol. (Amst.), 15, 247.

LEKSELl, L. (1939). Acta chir. scand., 82, 262.

MaCKenSen, G., and Harder, S. (1954). v. Graefes Arch. Ophthal., 155, 397.

Miles, W. R. (1939a). Proc. nat. Acad. Sci. (Wash.), 25, 128.

(1939b). Ibid., 25, 25.

(1939c). Yale J. Biol. Med., 12, 161.

(1940). Science, 91, 456.

Osiba, S. (1957). Jap. J. Physiol., 7, 355.

ShACKel, B. (1958). J. appl. Physiol., 13, 153.

(1959). Amer. J. Psychol., 72, 114

(1960). Brit. J. Ophthal., 44,89.

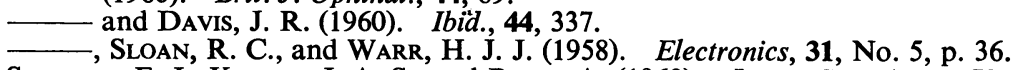

SWeEney, E. J., Kinney, J. A. S., and Ryan, A. (1960). J. opt. Soc. Amer., 50, 237-240.

Ten Doesschate, G., and Ten Doesschate, J. (1956). Ophthalmologica (Basel), 132, 308.

WhITFIELD, J. W. (1947). Biometrika, 34, 292. 